\title{
The genetic basis of idiopathic pulmonary fibrosis
}

\author{
Jonathan A. Kropski ${ }^{1}$, Timothy S. Blackwell ${ }^{1,2,3,4}$ and James E. Loyd ${ }^{1}$
}

Affiliations:

${ }^{1}$ Division of Allergy, Pulmonary and Critical Care Medicine, Dept of Medicine, Vanderbilt University School of Medicine, Nashville, TN, USA.

${ }^{2}$ Dept of Cell and Developmental Biology, Vanderbilt University School of Medicine, Nashville, TN, USA.

${ }^{3}$ Dept of Cancer Biology, Vanderbilt University School of Medicine, Nashville, TN, USA.

${ }^{4}$ Department of Veterans Affairs Medical Center, Nashville, TN, USA.

\section{Correspondence:}

Jonathan A. Kropski MD, Division of Allergy, Pulmonary and Critical Care Medicine, Dept of Medicine, Vanderbilt University School of Medicine, 1611 21st Avenue South, T-1218 Medical Center North, Nashville, TN 37232, USA.

Email: jon.kropskiđvanderbilt.edu

ABSTRACT Throughout the past decade, there have been substantial advances in understanding the pathogenesis of idiopathic pulmonary fibrosis (IPF). Recently, several large genome-wide association and linkage studies have identified common genetic variants in more than a dozen loci that appear to contribute to IPF risk. In addition, family-based studies have led to the identification of rare genetic variants in genes related to surfactant function and telomere biology, and mechanistic studies suggest pathophysiological derangements associated with these rare genetic variants are also found in sporadic cases of IPF. Current evidence suggests that rather than existing as distinct syndromes, sporadic and familial cases of IPF (familial interstitial pneumonia) probably reflect a continuum of genetic risk. Rapidly evolving bioinformatic and molecular biology techniques, combined with next-generation sequencing technologies, hold great promise for developing a comprehensive, integrated approach to defining the fundamental molecular mechanisms that underlie IPF pathogenesis.

@ERSpublications

Emerging genetic studies offer new insights into the fundamental mechanisms of pulmonary fibrosis http://ow.ly/KK9Q3

Editorial comment in Eur Respir J 2015; 45: 1539-1541 [DOI: 10.1183/09031936.00052715].

Received: Sept 042014 | Accepted after revision: March 17 2015 | First published online: April 022015

Conflict of interest: None declared.

Support statement: This study was funded by the US Department of Health and Human Services, National Institutes of Health (HL085317, HL092870, HL94296). Funding information for this article has been deposited with FundRef.

Copyright $\odot$ ERS 2015 


\section{Novel ideas}

Through the past decade, rapid advances in genetic and genomic technologies have begun to reshape our understanding of the "idiopathic" interstitial pneumonias. Genome-wide association studies have identified more than a dozen common genetic variants associated with idiopathic pulmonary fibrosis (IPF) risk, and may be linked to altered disease progression and survival. Rare genetic variants in eight genes have been implicated in familial interstitial pneumonia, the familial form of IPF, which broadly fall into two categories: genes related to surfactant protein processing and trafficking, and those linked to telomere biology. In addition to genetic links, unique disease phenotypes based on transcriptomic changes have been identified. As we go forward, we anticipate that advances in these genetic and genomic technologies will result in a re-organisation of the way we define and classify interstitial lung disease based on molecular characterisation. As we evolve from a system of diagnosis based on histopathology to one based on a specific genetic/genomic signature reflecting the fundamental biology of the disease, there will be unique opportunities to develop and test therapies in specific patient populations based on the molecular profiles. Coupled with advances in detection of early disease, the coming decade offers an unprecedented opportunity to dramatically change the lives of patients with IPF.

\section{Introduction}

Idiopathic pulmonary fibrosis (IPF), the most common of the idiopathic interstitial pneumonias (IIPs), is characterised by clinical symptoms of cough and dyspnoea, restrictive pulmonary function tests with impaired gas exchange, and progressive lung scarring [1]. Recently, two modestly effective drugs for treating IPF have been identified [2, 3]; however, the prognosis of IPF remains grave, emphasising a need for a more complete understanding of the mechanisms of disease pathogenesis. Available data indicate that both genetic and environmental factors contribute to the risk of IPF and other IIPs $[1,4,5]$. The first insights into IIP genetics came from studies of families with heritable cases of IIP, a syndrome termed familial interstitial pneumonia (FIP). As early as the 1950s, it was recognised that, on occasion, IIP cases clustered in families [6,7], suggesting a genetic basis to at least a subset of disease. By the 1990s, it was reported that FIP represented a rare subset of IIP, comprising 3-5\% of cases [8]. More recently, estimates from several independent groups have suggested that as many as $20 \%$ of IIP cases are familial [9-12]. Studies in families have uncovered rare genetic variants in eight genes that are linked to FIP, including three surfactant-related proteins (surfactant protein C, SFTPC [13-16]; surfactant protein A2, SFTPA2 [17]; and ATP-binding cassette member A3, ABCA3 [18, 19]) as well as five genes linked to telomere function (telomerase reverse transcriptase, TERT [20, 21]; human telomerase RNA component, $h T R$ [20, 21]; dyskerin, DKC1 [22-24]; telomere interacting factor 2, TINF2 [25-27]; and regulator of telomere elongation helicase, RTEL1 [28]). Rare genetic variants in FIP-associated genes can be found in some cases of sporadic IPF [12], and investigations into the mechanisms through which these mutated genes contribute to disease have uncovered common underlying pathobiological changes that probably contribute to progressive fibrotic remodelling in FIP and sporadic IPF [4, 5].

\section{Common genetic variants in IPF}

Several studies since the early 2000s have investigated the role of functional polymorphisms in a variety of genes in relationship to IPF risk (table 1). Variants in several genes related to inflammation and immune response, including transforming growth factor beta-1 (TGFB1) [44, 45], interleukin-1 receptor alpha (IL1RN) [29-31], interleukin 8 (IL8) [33], toll-like receptor 3 (TLR3) [34], HLA DRB1*1501 [37], as well as cell cycle progression related genes CDKN1A and TP53 [36], have been nominally associated with IPF risk or progression. However, results from these small studies have not yet been validated in independent cohorts. More recently, several large genome-wide linkage and association studies have been completed and have identified numerous additional loci that appear to confer risk for IPF.

\section{MUC5B}

In 2011, a genome-wide linkage study identified a locus on chromosome 11 that was significantly associated with IPF risk [38]. Resequencing of this region subsequently identified a common single nucleotide polymorphism (SNP) (rs35705950) in the promoter of the gene encoding for mucin 5B (Muc5B) that was associated with a six- to eight-fold increased risk for IPF. The association of this MUC5B promoter polymorphism and IPF has since been confirmed in several independent cohorts, predominantly in Caucasians [32, 39-42, 46]. Interestingly, it appears the MUC5B SNP has a similar frequency in FIP and sporadic IPF cases [38]. This association, however, may be specific to IIP among interstitial lung diseases (ILDs) since reports indicate rs35705950 does not confer increased risk of scleroderma-related ILD or sarcoidosis [39, 41, 47]. This association of rs35705950 with IPF was confirmed in a cohort of Mexican patients [48]; however, rs35705950 was found to be rare in a Korean 


\begin{tabular}{|c|c|c|c|c|c|}
\hline Locus & Gene & SNP & IPF risk & IPF survival & Reference \\
\hline \multirow[t]{3}{*}{$2 q 14$} & ILIRN & rs408392 & Yes & & [29-31] \\
\hline & & rs419598 & Yes & & \\
\hline & & rs2637988 & Yes & & \\
\hline $3 q 26$ & $h T R$ & rs6793295 & Yes & & [32] \\
\hline \multirow[t]{2}{*}{$4 q 13$} & IL8 & rs4073 & Yes & & [33] \\
\hline & & rs2227307 & Yes & & \\
\hline $4 q 22$ & FAM13A & rs2609255 & Yes & & [32] \\
\hline $4 q 35$ & TLR3 & rs3775291 & & Harmful & [34] \\
\hline $5 p 15$ & TERT & rs2736100 & Yes & & {$[32,35]$} \\
\hline $6 p 21$ & CDKN1A & rs2395655 & Yes & Harmful & [36] \\
\hline 6p21 & $H L A-D R B 1$ & & Yes & & [37] \\
\hline $6 q 24$ & $D S P$ & rs2076295 & Yes & & [32] \\
\hline $7 q 22$ & Intergenic & rs47274443 & Yes & & [32] \\
\hline $10 q 24$ & OBFC1 & rs11191865 & Yes & & [32] \\
\hline \multirow[t]{5}{*}{$11 \mathrm{p} 15$} & MUC5B & rs35705950 & Yes & Protective & {$[32,38-43]$} \\
\hline & MUC2 & rs7934606 & Yes & & [32] \\
\hline & TOLLIP & rs111521887 & Yes & & [40] \\
\hline & TOLLIP & rs5743894 & Yes & & {$[40]$} \\
\hline & TOLLIP & rs2743890 & Yes & Protective & [40] \\
\hline $13 q 34$ & ATP11A & rs1278769 & Yes & & [32] \\
\hline $14 q 21$ & MDGA2 & rs7144383 & Yes & & [40] \\
\hline $15 q 14-15$ & Intergenic & rs2034650 & Yes & & [32] \\
\hline \multirow[t]{2}{*}{$17 q 13$} & TP53 & rs12951053 & No & Harmful & {$[36]$} \\
\hline & TP53 & rs12602273 & No & Harmful & \\
\hline $17 q 21$ & MAPT & rs1981997 & Yes & & [32] \\
\hline $17 q 21$ & SPPL2C & rs17690703 & Yes & & [40] \\
\hline $19 q 13$ & $D P P 9$ & rs12610495 & Yes & & [32] \\
\hline $19 q 13$ & TGFB1 & rs1800470 & No & Harmful & {$[44,45]$} \\
\hline
\end{tabular}

cohort of IPF patients. Similarly, in a Chinese population, rs35705950 was rare in IPF patients but different MUC5B polymorphisms were associated with disease [49].

While the rs35705950 MUC5B SNP was associated with increased MUC5B mRNA expression in lungs of control subjects, MUC5B expression was uniformly increased in lungs of IPF patients compared to controls, regardless of whether the $M U C 5 B$ SNP was present [38]. Consistent with this observation, increased numbers of $M U C 5 B$ expressing cells have been detected in the distal airways of IPF patients [50]. MUC5B rs35705950 has also been reported as a risk factor for asymptomatic interstitial lung abnormalities detected on computed tomography (CT) scan among subjects over the age of 50 years in the Framingham cohort [51]. Surprisingly, although minor allele carriers of rs35705950 have increased risk of developing disease, IPF patients who carry the minor (risk) allele appear to have improved survival compared to noncarriers [43]. Previous animal studies have suggested that MUC5B regulates airway host defence [52], but the mechanisms by which MUC5B influences fibrotic remodelling are uncertain at present.

Insights from genome-wide association studies

A major advance of the past several years has been the development of large, robust datasets with sufficient statistical power for genome-wide association studies. Two large independent genome-wide association studies of IPF patients have now been conducted and identified numerous genetic loci that confer IPF risk. The first, published in 2013 [32], evaluated 1616 IIP cases (the vast majority of which were IPF) and 4683 control subjects with replication in an additional 876 cases and 1890 controls. In addition to confirming the previously reported association with $M U C 5 B$, nine additional loci were significantly associated with IIP, predominantly IPF (table 1), including SNPs near TERT and hTR. 10 SNPs on chromosome $11 \mathrm{p} 15$ nominally met genome-wide significance, but after controlling for MUC5B rs35705950 these loci no longer met genome-wide significance, suggesting that weak linkage disequilibrium with the MUC5B promoter polymorphism was largely responsible for this association.

Results of a second genome-wide association study again implicated a locus on chromosome 11p15 as significantly associated with IPF [40], but did not replicate other risk loci identified by FINGERLIN et al. [32]. This genome-wide association study performed a three-stage analysis, including a discovery and two replication cohorts, comprising in total 1410 IPF cases and 2934 control individuals. Five loci achieved 
genome-wide significance, including four SNPs on chromosome 11p15 and one on 17q21. Among the 11 p15 SNPs were MUC5B rs35705950 and three SNPs within the Toll-interacting protein (TOLLIP) locus. Linkage disequilibrium was reported to be low with rs35705950, suggesting TOLLIP may represent an independent risk locus. Similar to MUC5B rs35705950, IPF cases with the TOLLIP risk allele (the major allele) had decreased mortality compared to minor allele carriers.

Deciphering the biological effects of common genetic variants identified by genome-wide association studies has proven challenging so far. It is possible that the relevant biological effect of most individual SNPs is subtle or manifests only in the context of unique additional genetic or environmental factors to confer disease risk. Despite challenges, future studies are needed to clarify the biological role of disease-associated common genetic variants.

\section{Rare genetic variants in FIP and IPF}

FIP and sporadic IPF share many clinical and histopathological features [53], which has led to the hypothesis that similar mechanisms underlie the pathogenesis of sporadic and familial disease. Additionally, ScHOLAND et al. [54] employed an extensive genealogical database and found unexpected relatedness among patients who died of what was believed to be sporadic IPF, further supporting the idea that the genetic landscapes of sporadic IPF and FIP overlap considerably. Together, studies to date suggest that sporadic and familial disease reflect of spectrum of genetic risk for pulmonary fibrosis (fig. 1). In this model, genetic risk factors of small and large effects interact with rare and common environmental stimuli to produce the phenotype of pulmonary fibrosis. Most FIP kindreds appear to have an autosomal dominant inheritance pattern with incomplete penetrance, suggesting an important role for genetic rare variants of large effect. In contrast, sporadic IPF may occur more often in the setting of de novo or low penetrance rare variants, or a combination of more common, less severe genetic risk alleles. As described below, in some cases genetic rare variants of large effect may be found in genes that lie within loci also containing common variants associated with IPF risk. Focusing on familial disease offers the ability to use Mendelian approaches to identify disease-associated rare variants. This represents a promising approach to enhance mechanistic understanding of the impact of genetic risk factors on development of FIP and potentially sporadic IPF.

\section{Telomerase and short telomeres}

Pulmonary fibrosis occurs in approximately $20 \%$ of patients with dyskeratosis congenita [55], a rare inherited genetic disorder characterised by leukoplakia, bone marrow failure and dystrophic nails that typically affects young males. Rare variants in genes related to telomere biology have been implicated in dyskeratosis congenita [56]. In 2007, using candidate gene approaches, two groups identified heterozygous loss-of-function rare variants in telomere-related genes in $7-15 \%$ of FIP families who did not have a history of dyskeratosis congenita [20,21]. These variants in TERT and $h T R$ lead to short telomeres in peripheral blood and in the lung $[20,21,57,58]$. To date, TERT rare variants are the most commonly identified mutations linked to FIP; however, TERT rare variants are rarely identified in sporadic cases of IPF [57]. In addition to variants in TERT and $h T R$, two recent reports identified FIP patients with rare variants in the gene encoding for dyskerin (DKC1) [22, 23], another component of the telomerase complex. Several reports have also identified pulmonary fibrosis in families with dyskeratosis congenita associated with rare variants in TINF2 [26, 27]. In one of these families, there was evidence of somatic or acquired mosaicism for a deletion which abolished expression of the missense variant [25]. This interesting observation suggests acquired genetic variation may represent one mechanism regulating the clinical spectrum of disease linked to telomere pathway rare variants.

Using whole-exome sequencing in a cohort of $>180$ FIP kindreds, our group has identified heterozygous loss-of-function rare variants in another telomere-related gene, regulator of telomere elongation helicase (RTEL1) in nine families with IPF [28]. Similar to TERT, hTR and DKC1 rare variants, these RTEL1 rare variants are associated with short telomeres in peripheral blood. In addition to a role in telomere

Sporadic idiopathic pulmonary fibrosis

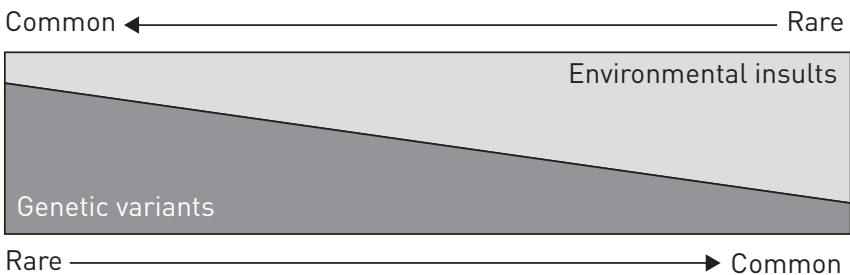

Familial interstitial pneumonia
FIGURE 1 Proposed model of geneenvironment interactions in the pathogenesis of pulmonary fibrosis. 
maintenance, RTEL1 appears to play a more general role in genome stability, DNA repair and replication [59-61], suggesting it may confer disease risk through additional mechanisms. As TERT deficiency has also been associated with abnormal DNA repair [62], it is possible that this mechanism, rather than having a direct effect on telomere length, could be an important in mediating disease risk associated with telomerase pathway rare variants. Cumulatively, rare variants in these five telomere-related genes are reported in approximately $15-20 \%$ of FIP families.

Notably, the short telomere phenotype in peripheral blood mononuclear cells (PBMCs) is not limited to patients with loss of function rare variants in telomerase complex genes. Approximately one-third of sporadic IPF and FIP patients have short telomeres $(<10$ th percentile for age) in PBMCs [57, 58]. In addition, it appears that the majority of IPF patients have short telomeres in alveolar epithelial cells [23, 57], suggesting that additional factors (besides genetic risk) contribute to telomere shortening in lungs of patients with IPF and FIP. Interestingly, asymptomatic first-degree relatives of FIP patients have decreased alveolar epithelial cell telomere length compared to controls, and alveolar epithelial cell telomere length is significantly associated with the presence of interstitial changes on high-resolution chest CT [63]. In addition, it appears that PBMC telomere length within families with TERT rare variants can be inherited, at least in part independently of a known rare variant, producing a unique scenario of inherited genetic risk without the risk allele $[21,57,64]$. PBMC telomere length appears to be predictive of survival among patients with IPF, wherein IPF patients with short telomeres have reduced survival compared to those with "normal" length PBMC telomeres [65]. In addition to rare genetic variants in telomere related genes, common genetic variants in loci near TERT, $h T R$ and telomere gene OBFC1 have been linked to sporadic IPF by a genome-wide association study [32] and may be an important factor in determining telomere length. Environmental factors, including cigarette smoke exposure, may also play a role in telomere shortening in FIP and sporadic IPF [66].

Genetic and clinical evidence provide a compelling association between lung fibrosis and telomere biology. Although the mechanisms through which telomerase pathway rare variants lead to lung fibrosis are uncertain, it has been suggested that these loss of function variants disrupt lung epithelial repair mechanisms [67]. Murine models of telomerase dysfunction have been developed but present a number of challenges that limit their utility for mechanistic studies [4]. In spite of these limitations, several studies have reported attempts to model telomerase deficiency in the lung. Tert null mice have decreased numbers of alveolar epithelial cells and modest architectural changes in the lung [68]. These mice have increased susceptibility to cigarette smoke induced-emphysema [69], but do not develop lung fibrosis. Studies using pro-fibrotic stimuli such as bleomycin to investigate fibrotic susceptibility in Tert and Terc null mice have yielded conflicting results $[70,71]$. Together, it appears that recapitulating the biology of telomere dysfunction in humans using mouse models is problematic. Therefore, new approaches are needed in this area.

\section{Surfactant protein-related genes}

NogeE et al. [13] first described a heterozygous mutation in the gene encoding surfactant protein C (SFTPC) in a young woman and her child with IIP in 2001. Soon after, we identified the first association between SFTPC and FIP in a large family with 11 affected individuals [14]. Subsequently, other groups have reported heterozygous rare variants in SFTPC in 1-2\% of FIP [9, 12, 14-16, 72]. Although one group reported SFTPC rare variants in $25 \%$ of FIP kindreds [15], this high frequency was probably related to founder effects. The mechanisms through which SFTPC rare variants contribute to disease pathogenesis have recently been reviewed elsewhere [4, 73]. In brief, it appears that C-teminal BRICHOS domain mutants result in defects in folding of the propeptide within the endoplasmic reticulum, leading to endoplasmic reticulum stress and activation of the unfolded protein response [74-78]. Linker domain mutants (such as the I73T rare variant) appear to alter trafficking of the pro-peptide [79] and lead to dysregulated proteastasis [80]. Animal modelling suggests induction of endoplasmic reticulum stress in alveolar epithelial cells is not sufficient to induce spontaneous fibrosis but results in an exaggerated fibrotic response following low-dose bleomycin challenge [81]. Induction of endoplasmic reticulum stress in alveolar epithelial cells increases susceptibility to apoptotic stimuli [81, 82], increases expression of mesenchymal markers, and enhances production of profibrotic mediators [83, 84]. In addition, rare variants in another surfactant protein (SFTPA2) [17] has been linked to FIP. SFTPA2 rare variants also result in endoplasmic reticulum stress and may increase latent TGF $\beta$ activation $[85,86]$.

While the frequency of surfactant protein rare variants in sporadic IPF appears to be low [87, 88], several groups have reported that endoplasmic reticulum stress and unfolded protein response activation is a common feature of FIP and sporadic IPF [75, 89], suggesting that environmental factors (such as herpesviruses [75, 90] and tobacco smoke [91-93]) may contribute to this phenotype. Promisingly, it has recently been shown that pharmacological chaperones might improve processing of mutant surfactant 
proteins in alveolar epithelial cell lines [94]. These exciting developments raise the possibility of targeted therapies for at least a subset of patients with pulmonary fibrosis.

In addition, rare variants in another gene involved in surfactant processing, ATP-binding cassette-type 3 $(A B C A 3)$ (previously linked to paediatric interstitial lung disease) have been reported in several FIP families [18, 19, 95], as well as in sporadic cases of IPF [12]. In one consanguineous family [18], homozygous rare variants in $A B C A 3$ were identified. A heterozygous rare variant in $A B C A 3$ was also reported in a patient with "combined pulmonary fibrosis and emphysema" [19]. In another family carrying the I73T SFTPC rare variant, a second heterozygous rare variant in $A B C A 3$ modified disease penetrance [95]. The exact mechanisms by which $A B C A 3$ variants confer FIP risk are unclear at present, but presumably relate to epithelial cell dysfunction.

\section{ELMOD2}

A report using linkage in a cohort of Finnish FIP families suggested ELMOD2 as a candidate FIP gene [96] and in vitro studies suggest ELMOD2 may play a role in antiviral responses [97]. Whether ELMOD2 plays an important role in lung fibrosis has not yet been elucidated.

\section{Missing heritability and future genetic discovery}

Cumulatively, available literature suggests that rare variants in FIP genes SFTPC, SFTPA2, ABCA3, TERT, $h T R, D K C 1$, TINF2 and RTEL1 comprise 15-20\% of FIP cases (table 2). However, it is possible that selection of families for these candidate-based genetic studies, as well as founder effects in certain populations, may have overestimated the frequency of some variants among all FIP families. While common genetic variants also confer FIP risk and may explain as much as $30 \%$ of FIP risk [32], there remains substantial "missing heritability". In an effort to identify novel FIP genes, recently, our group has performed extensive whole-exome sequencing of subjects from FIP kindreds. While this approach has implicated RTEL1 as an FIP gene, ongoing analysis suggests that rare variants in a single gene (or small group of related genes) do not account for disease in a majority of families [28]. Compared to other genetic lung diseases, such as cystic fibrosis and familial pulmonary arterial hypertension, it appears the genetic basis of FIP is substantially more heterogenous. Given the lack of a dominant gene in FIP, the principal challenge is one of power, both within families and across subjects. Linkage or co-segregation based approaches rely upon the power of large, multigenerational pedigrees with numbers of affected individuals, a well-established inheritance model, and ability to clearly ascertain affection status. Considering that each individual typically harbours 200 rare genetic variants in their exomes [98] and most FIP kindreds are small, dozens of rare variants will be shared among affected individuals, making it difficult to identify the culprit rare variant by this approach. In light of what appears to be substantial allelic heterogeneity, similar to what has been observed for other familial disorders including hypercholesterolaemia [99], neurodegenerative diseases [100] and cardiomyopathies [101], functional testing and validation in cell and/or animal models will be critical to determine the pathogenicity of specific variants and genes tentatively linked to disease.

These challenges suggest that novel and creative approaches will be necessary to further genetic discovery in FIP and IPF. As elaborated further below, we anticipate that evolving bioinformatic approaches to variant prioritisation [102, 103], coupled with network- and pathway-based analysis [104] hold promise for identifying, validating and integrating disease-associated variants. In addition to rare coding variants, future studies investigating intronic variants or variants in more distant cis- and trans- regulatory regions should further inform understanding of the spectrum of genetic risk for FIP.

\begin{tabular}{lcc}
$\begin{array}{l}\text { TABLE } 2 \text { Rare genetic variants linked to familial interstitial pneumonia (FIP) } \\
\text { Gene }\end{array}$ & Reported \% of FIP & Reference \\
\hline TERT & $8-15 \%$ & {$[20,21]$} \\
RTEL1 & $5 \%$ & {$[28]$} \\
hTR & $<1 \%$ & {$[20,21]$} \\
DKC1 & $<1 \%$ & {$[23,24]$} \\
TINF2 & $<1 \%$ & {$[25-27]$} \\
SFTPC & $2-25 \%$ & {$[13-16,87,88]$} \\
SFTPA2 & $<1 \%$ & {$[17]$} \\
ABCA3 & $<1 \%$ & {$[18,19]$} \\
Unknown & $75-85 \%$ &
\end{tabular}




\section{Implications for other IIPs}

The evolving understanding of the genetic landscape of FIP and sporadic IPF leads to the question of whether the same genes and genetic variants confer risk for other ILDs, including other forms of IIP. Genetic predisposition for IIPs other than IPF has been poorly characterised; however, several clues suggest there are both conserved and distinct genetic risk factors. Within FIP pedigrees, it has been well recognised that individuals may harbour the same disease-associated rare variant yet present with different histopathologies $[13,53]$. This finding indicates that differential environmental exposures overlaid on a common set of genetic risk factors may play a role in determining IIP phenotype. No study to date has been adequately powered to assess whether common genetic variants linked to IPF also confer risk to other IIPs. Future studies are required to address this important issue.

\section{Implications for clinical management \\ Impact on clinical trial design and treatment}

Although no clinical trials to date have stratified patients based on genetic risk, this strategy could prove useful in light of the now recognised associations of the $11 \mathrm{p} 15$ risk variants (MUC5B and TOLLIP) with reduced disease progression and improved survival. As additional genes and risk alleles are identified, more complex stratification schemes may be developed to enhance study design. With the recent publication of randomised controlled trials of two pharmacological agents, pirfenidone [2] and nintedanib [3], that reduce lung function decline among IPF patients, a reasonable question for future study is whether genetic factors influence response to treatment with one or both of these medications.

Whether genetic factors influence outcomes after lung transplant remains an underexplored question. One small series described successful lung transplant in eight patients with TERT rare variants, but a high rate of haematological and renal toxicities were noted [105]. The role of other rare or common genetic variants on outcome after lung transplant is not known.

\section{Genetic testing}

As our understanding of the influence of genetic factors on risk of IPF and its natural history, a potential role for clinical genetic testing emerges. At present, we suggest that evidence is not sufficient to recommend routine genetic testing for rare or common genetic variants for patients with sporadic IPF. In families with FIP, clinical testing for variants in SFTPC, SFTPA2, and telomerase-related genes including TERT, $h T R$, $D K C 1$ and RTEL1 is available from commercial and academic sources. Our practice is to offer genetic counselling and consideration of genetic testing to patients with FIP and a family history suggestive of a telomerase dysfunction syndrome (including diagnoses of aplastic anaemia, cryptogenic cirrhosis or premature graying). Decisions to undergo genetic testing are complex and highly individual, and no studies have evaluated the impact of genetic testing on patients/families in the context of FIP. Extrapolating from our experience and literature from other disorders [106-108], continued close follow-up of patients who undergo genetic testing appears essential regardless of whether they are found to carry a disease-associated rare variant. In light of the incomplete penetrance and variable expressivity of FIP-associated rare variants, we recommend patients and their families confer with genetic counsellors before consideration of genetic tests that are currently available or may become available in the future. Over time, we anticipate there may be a role for broader screening of common and rare genetic variants associated with IPF.

\section{Overcoming challenges and future directions}

To date, investigations of the genetic basis of FIP and sporadic IPF have provided crucial insights into underlying mechanisms of progressive pulmonary fibrosis. We suggest that there is probably not a single "road to IPF" but rather there are "multiple paths" that converge to a common phenotype. The development of exciting next-generation sequencing capabilities, along with continuously evolving bioinformatic approaches to analysis of large datasets and rapidly improving molecular biological techniques, offer unique possibilities to identify additional novel genes and pathways that contribute to the pathogenesis of progressive pulmonary fibrosis (fig. 2). Whole-genome sequencing is rapidly becoming feasible, and although it presents new and greater bioinformatic challenges, the possibility of analysing noncoding variants, as well as interactions among variants, holds promise in identifying as yet unexplained heritability of FIP and sporadic IPF. In addition, the rapidly evolving field of stem cell biology offers the possibility of studying the effects of genetic variants in primary human cell types of interest using inducible pluripotent stem cell differentiation strategies $[109,110]$. The development of improved gene editing technologies, including the CRISPR-Cas9-based system, should facilitate enhanced ability to characterise genetic variants with functional testing in vitro and in vivo [111, 112].

We anticipate that by understanding the biological mechanisms through which individual genetic variants contribute to disease pathogenesis, key pathways will be identified that will clarify the crucial molecular mediators of IPF pathogenesis. We anticipate that a role for molecular genetics in the classification of IIPs 


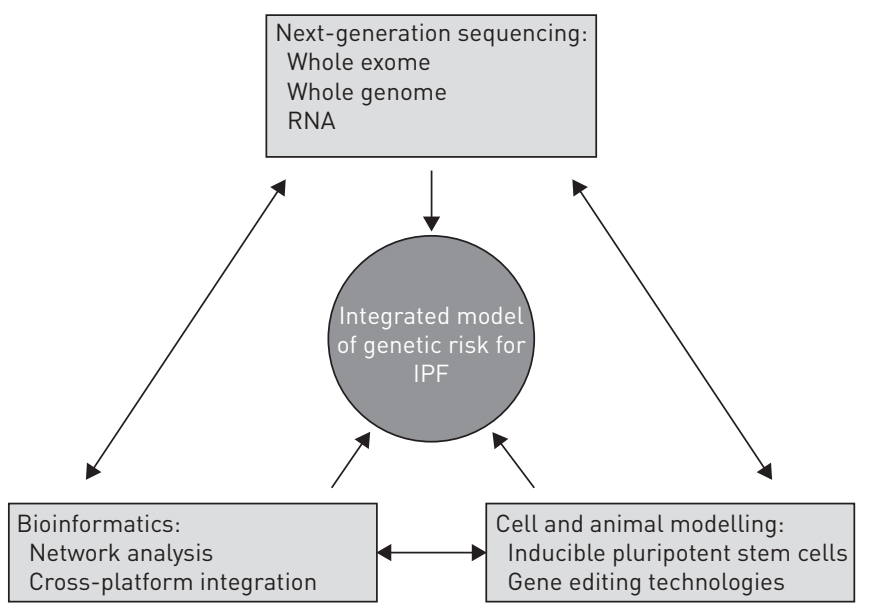

FIGURE 2 Paradigm to develop an integrated model of genetic risk for idiopathic pulmonary fibrosis (IPF). Future studies identifying and characterising the role of genetic variants in IPF will require integration of next-generation sequencing technologies, bioinformatics, and use of state-of-the-art molecular biology in cell and animal models. Identification of variants by whole exome sequencing or whole genome sequencing will require strategic bioinformatics approaches. These genetic variants will require functional validation in cell and animal models; characterising the effects of these genetic variants on gene expression profiles will require integration of sequencing and bioinformatics technologies.

will emerge. The ultimate challenge that lies ahead is to develop an integrated understanding of the role of genetic variants (rare and common) and to identify how these variants interact with each other and with environmental factors to produce the epigenetic, transcriptomic, proteomic, histopathologic, and clinical features of IPF. With increased understanding of the fundamental mechanisms of disease, the future is promising for development of new, targeted therapies to further improve treatment of IPF.

\section{References}

$1 \quad$ King TE Jr, Pardo A, Selman M. Idiopathic pulmonary fibrosis. Lancet 2011; 378: 1949-1961.

2 King TE Jr, Bradford WZ, Castro-Bernardini S, et al. A phase 3 trial of pirfenidone in patients with idiopathic pulmonary fibrosis. N Engl J Med 2014; 370: 2083-2092.

3 Richeldi L, du Bois RM, Raghu G, et al. Efficacy and safety of nintedanib in idiopathic pulmonary fibrosis. N Engl J Med 2014; 370: 2071-2082.

4 Kropski JA, Lawson WE, Young LR, et al. Genetic studies provide clues on the pathogenesis of idiopathic pulmonary fibrosis. Dis Model Mech 2013; 6: 9-17.

5 Spagnolo P, Grunewald J, du Bois RM. Genetic determinants of pulmonary fibrosis: evolving concepts. Lancet Respir Med 2014; 2: 416-428.

6 Bonanni PP, Frymoyer JW, Jacox RF. A family study of idiopathic pulmonary fibrosis. a possible dysproteinemic and genetically determined disease. Am J Med 1965; 39: 411-421.

7 Peabody JW, Peabody JW Jr, Hayes EW, et al. Idiopathic pulmonary fibrosis; its occurrence in identical twin sisters. Dis Chest 1950; 18: 330-344.

8 Hodgson U, Laitinen T, Tukiainen P. Nationwide prevalence of sporadic and familial idiopathic pulmonary fibrosis: evidence of founder effect among multiplex families in Finland. Thorax 2002; 57: 338-342.

9 Fernandez BA, Fox G, Bhatia R, et al. A Newfoundland cohort of familial and sporadic idiopathic pulmonary fibrosis patients: clinical and genetic features. Respir Res 2012; 13: 64.

10 Garcia-Sancho C, Buendia-Roldan I, Fernandez-Plata MR, et al. Familial pulmonary fibrosis is the strongest risk factor for idiopathic pulmonary fibrosis. Respir Med 2011; 105: 1902-1907.

11 Loyd JE. Pulmonary fibrosis in families. Am J Respir Cell Mol Biol 2003; 29: Suppl. 3, S47-S50.

12 Coghlan MA, Shifren A, Huang HJ, et al. Sequencing of idiopathic pulmonary fibrosis-related genes reveals independent single gene associations. BMJ Open Respir Res 2014; 1: e000057.

13 Nogee LM, Dunbar AE 3rd, Wert SE, et al. A mutation in the surfactant protein C gene associated with familial interstitial lung disease. N Engl J Med 2001; 344: 573-579.

14 Thomas AQ, Lane K, Phillips J 3rd , et al. Heterozygosity for a surfactant protein $\mathrm{C}$ gene mutation associated with usual interstitial pneumonitis and cellular nonspecific interstitial pneumonitis in one kindred. Am J Respir Crit Care Med 2002; 165: 1322-1328.

15 van Moorsel $\mathrm{CH}$, van Oosterhout MF, Barlo NP, et al. Surfactant protein C mutations are the basis of a significant portion of adult familial pulmonary fibrosis in a Dutch cohort. Am J Respir Crit Care Med 2010; 182: 1419-1425.

16 Ono S, Tanaka T, Ishida M, et al. Surfactant protein C G100S mutation causes familial pulmonary fibrosis in Japanese kindred. Eur Respir J 2011; 38: 861-869.

17 Wang Y, Kuan PJ, Xing C, et al. Genetic defects in surfactant protein A2 are associated with pulmonary fibrosis and lung cancer. Am J Hum Genet 2009; 84: 52-59.

18 Campo I, Zorzetto M, Mariani F, et al. A large kindred of pulmonary fibrosis associated with a novel ABCA3 gene variant. Respir Res 2014; 15: 43. 
Epaud R, Delestrain C, Louha M, et al. Combined pulmonary fibrosis and emphysema syndrome associated with ABCA3 mutations. Eur Respir J 2014; 43: 638-641.

Armanios MY, Chen JJ, Cogan JD, et al. Telomerase mutations in families with idiopathic pulmonary fibrosis. N Engl J Med 2007; 356: 1317-1326.

Tsakiri KD, Cronkhite JT, Kuan PJ, et al. Adult-onset pulmonary fibrosis caused by mutations in telomerase. Proc Natl Acad Sci USA 2007; 104: 7552-7557.

Hisata S, Sakaguchi H, Kanegane H, et al. A novel missense mutation of DKC1 in dyskeratosis congenita with pulmonary fibrosis. Sarcoidosis Vasc Diffuse Lung Dis 2013; 30: 221-225.

Kropski JA, Mitchell DB, Markin C, et al. A novel dyskerin (DKC1) mutation is associated with familial interstitial pneumonia. Chest 2014; 146: e1-e7.

Alder JK, Parry EM, Yegnasubramanian S, et al. Penetrant telomere phenotypes in females with heterozygous mutations in the dyskeratosis congenita 1 (DKC1) gene. Hum Mutat 2013; 34: 1481-1485.

Alder JK, Stanley SE, Wagner CL, et al. Exome sequencing identifies mutant TINF2 in a family with pulmonary fibrosis. Chest 2014 [in press; DOI: 10.1378/chest.14-1947].

Fukuhara A, Tanino Y, Ishii T, et al. Pulmonary fibrosis in dyskeratosis congenita with TINF2 gene mutation. Eur Respir J 2013; 42: 1757-1759.

Kannengiesser C, Borie R, Revy P. Pulmonary fibrosis associated with TINF2 gene mutation: is somatic reversion required? Eur Respir J 2014; 44: 269-270.

Cogan JD, Kropski JA, Zhao M, et al. Rare variants in RTEL1 are associated with familial interstitial pneumonia. Am J Respir Crit Care Med 2015; 191: 646-655.

Whyte M, Hubbard R, Meliconi R, et al. Increased risk of fibrosing alveolitis associated with interleukin-1 receptor antagonist and tumor necrosis factor-alpha gene polymorphisms. Am J Respir Crit Care Med 2000; 162: 755-758.

Barlo NP, van Moorsel CH, Korthagen NM, et al. Genetic variability in the IL1RN gene and the balance between interleukin (IL)-1 receptor agonist and IL-1beta in idiopathic pulmonary fibrosis. Clin Exp Immunol 2011; 166: 346-351.

Korthagen NM, van Moorsel CH, Kazemier KM, et al. IL1RN genetic variations and risk of IPF: a meta-analysis and mRNA expression study. Immunogenetics 2012; 64: 371-377.

Fingerlin TE, Murphy E, Zhang W, et al. Genome-wide association study identifies multiple susceptibility loci for pulmonary fibrosis. Nat Genet 2013; 45: 613-620.

Ahn MH, Park BL, Lee SH, et al. A promoter SNP rs4073T>A in the common allele of the interleukin 8 gene is associated with the development of idiopathic pulmonary fibrosis via the IL-8 protein enhancing mode. Respir Res 2011; 12: 73 .

O'Dwyer DN, Armstrong ME, Trujillo G, et al. The Toll-like receptor 3 L412F polymorphism and disease progression in idiopathic pulmonary fibrosis. Am J Respir Crit Care Med 2013; 188: 1442-1450.

Mushiroda T, Wattanapokayakit S, Takahashi A, et al. A genome-wide association study identifies an association of a common variant in TERT with susceptibility to idiopathic pulmonary fibrosis. J Med Genet 2008; 45: 654-656.

Korthagen NM, van Moorsel CH, Barlo NP, et al. Association between variations in cell cycle genes and idiopathic pulmonary fibrosis. PLoS One 2012; 7: e30442.

Xue J, Gochuico BR, Alawad AS, et al. The HLA class II Allele DRB1*1501 is over-represented in patients with idiopathic pulmonary fibrosis. PLoS One 2011; 6: el4715.

Seibold MA, Wise AL, Speer MC, et al. A common MUC5B promoter polymorphism and pulmonary fibrosis. N Engl J Med 2011; 364: 1503-1512.

Borie R, Crestani B, Dieude P, et al. The MUC5B variant is associated with idiopathic pulmonary fibrosis but not with systemic sclerosis interstitial lung disease in the European Caucasian population. PLoS One 2013; 8: e70621.

Noth I, Zhang Y, Ma SF, et al. Genetic variants associated with idiopathic pulmonary fibrosis susceptibility and mortality: a genome-wide association study. Lancet Respir Med 2013; 1: 309-317.

Peljto AL, Steele MP, Fingerlin TE, et al. The pulmonary fibrosis-associated MUC5B promoter polymorphism does not influence the development of interstitial pneumonia in systemic sclerosis. Chest 2012; 142: 1584-1588.

Zhang Y, Noth I, Garcia JG, et al. A variant in the promoter of MUC5B and idiopathic pulmonary fibrosis. N Engl J Med 2011; 364: 1576-1577.

Pelito AL, Zhang Y, Fingerlin TE, et al. Association between the MUC5B promoter polymorphism and survival in patients with idiopathic pulmonary fibrosis. JAMA 2013; 309: 2232-2239.

Son JY, Kim SY, Cho SH, et al. TGF-betal T869C polymorphism may affect susceptibility to idiopathic pulmonary fibrosis and disease severity. Lung 2013; 191: 199-205.

Xaubet A, Marin-Arguedas A, Lario S, et al. Transforming growth factor-betal gene polymorphisms are associated with disease progression in idiopathic pulmonary fibrosis. Am J Respir Crit Care Med 2003; 168: 431-435.

Horimasu Y, Ohshimo S, Bonella F, et al. MUC5B promoter polymorphism in Japanese patients with idiopathic pulmonary fibrosis. Respirology 2015; 20: 439-444.

Stock CJ, Sato H, Fonseca C, et al. Mucin 5B promoter polymorphism is associated with idiopathic pulmonary fibrosis but not with development of lung fibrosis in systemic sclerosis or sarcoidosis. Thorax 2013; 68: 436-441.

Peljto AL, Selman M, Kim DS, et al. The MUC5B promoter polymorphism is associated with IPF in a Mexican cohort but is rare among Asian ancestries. Chest 2015; 147: 460-464.

Wei R, Li C, Zhang M, et al. Association between MUC5B and TERT polymorphisms and different interstitial lung disease phenotypes. Transl Res 2014; 163: 494-502.

Seibold MA, Smith RW, Urbanek C, et al. The idiopathic pulmonary fibrosis honeycomb cyst contains a mucocilary pseudostratified epithelium. PLoS One 2013; 8: e58658.

Hunninghake GM, Hatabu $\mathrm{H}$, Okajima $\mathrm{Y}$, et al. MUC5B promoter polymorphism and interstitial lung abnormalities. N Engl J Med 2013; 368: 2192-2200.

Roy MG, Livraghi-Butrico A, Fletcher AA, et al. Muc5b is required for airway defence. Nature 2014; 505: $412-416$.

Steele MP, Speer MC, Loyd JE, et al. Clinical and pathologic features of familial interstitial pneumonia. Am Respir Crit Care Med 2005; 172: 1146-1152. 
54

Scholand MB, Coon H, Wolff R, et al. Use of a genealogical database demonstrates heritability of pulmonary fibrosis. Lung 2013; 191: 475-481.

55 Armanios M. Telomerase and idiopathic pulmonary fibrosis. Mutat Res 2012; 730: 52-58.

56 Calado RT, Young NS. Telomere diseases. N Engl J Med 2009; 361: 2353-2365.

57 Alder JK, Chen JJ, Lancaster L, et al. Short telomeres are a risk factor for idiopathic pulmonary fibrosis. Proc Natl Acad Sci USA 2008; 105: 13051-13056.

58 Cronkhite JT, Xing C, Raghu G, et al. Telomere shortening in familial and sporadic pulmonary fibrosis. Am J Respir Crit Care Med 2008; 178: 729-737.

59 Uringa EJ, Lisaingo K, Pickett HA, et al. RTEL1 contributes to DNA replication and repair and telomere maintenance. Mol Biol Cell 2012; 23: 2782-2792.

60 Vannier JB, Sandhu S, Petalcorin MI, et al. RTEL1 is a replisome-associated helicase that promotes telomere and genome-wide replication. Science 2013; 342: 239-242.

61 Vannier JB, Sarek G, Boulton SJ. RTEL1: functions of a disease-associated helicase. Trends Cell Biol 2014; 24: $416-425$.

62 Masutomi K, Possemato R, Wong JM, et al. The telomerase reverse transcriptase regulates chromatin state and DNA damage responses. Proc Natl Acad Sci USA 2005; 102: 8222-8227.

63 Kropski JA, Pritchett JM, Zoz DF, et al. Extensive phenotyping of individuals at-risk for familial interstitial pneumonia reveals clues to the pathogenesis of interstitial lung disease. Am J Respir Crit Care Med 2015; 191: 417-427.

64 Diaz de Leon A, Cronkhite JT, Katzenstein AL, et al. Telomere lengths, pulmonary fibrosis and telomerase (TERT) mutations. PLoS One 2010; 5: e10680.

65 Stuart BD, Lee JS, Kozlitina J, et al. Effect of telomere length on survival in patients with idiopathic pulmonary fibrosis: an observational cohort study with independent validation. Lancet Respir Med 2014; 2: 557-565.

66 Theall KP, McKasson S, Mabile E, et al. Early hits and long-term consequences: tracking the lasting impact of prenatal smoke exposure on telomere length in children. Am J Public Health 2013; 103: Suppl. 1, S133-S135.

67 Driscoll B, Buckley S, Bui KC, et al. Telomerase in alveolar epithelial development and repair. Am J Physiol Lung Cell Mol Physiol 2000; 279: L1191-L1198.

68 Lee J, Reddy R, Barsky L, et al. Lung alveolar integrity is compromised by telomere shortening in telomerase-null mice. Am J Physiol Lung Cell Mol Physiol 2009; 296: L57-L70.

69 Alder JK, Guo N, Kembou F, et al. Telomere length is a determinant of emphysema susceptibility. Am J Respir Crit Care Med 2011; 184: 904-912.

70 Degryse AL, Xu XC, Newman JL, et al. Telomerase deficiency does not alter bleomycin-induced fibrosis in mice. Exp Lung Res 2012; 38: 124-134.

71 Liu T, Chung MJ, Ullenbruch M, et al. Telomerase activity is required for bleomycin-induced pulmonary fibrosis in mice. J Clin Invest 2007; 117: 3800-3809.

72 Cottin V, Reix P, Khouatra C, et al. Combined pulmonary fibrosis and emphysema syndrome associated with familial SFTPC mutation. Thorax 2011; 66: 918-919.

73 Tanjore H, Blackwell TS, Lawson WE. Emerging evidence for endoplasmic reticulum stress in the pathogenesis of idiopathic pulmonary fibrosis. Am J Physiol Lung Cell Mol Physiol 2012; 302: L721-L729.

74 Bridges JP, Wert SE, Nogee LM, et al. Expression of a human surfactant protein C mutation associated with interstitial lung disease disrupts lung development in transgenic mice. J Biol Chem 2003; 278: 52739-52746.

75 Lawson WE, Crossno PF, Polosukhin VV, et al. Endoplasmic reticulum stress in alveolar epithelial cells is prominent in IPF: association with altered surfactant protein processing and herpesvirus infection. Am J Physiol Lung Cell Mol Physiol 2008; 294: L1119-L1126.

76 Mulugeta S, Maguire JA, Newitt JL, et al. Misfolded BRICHOS SP-C mutant proteins induce apoptosis via caspase-4- and cytochrome c-related mechanisms. Am J Physiol Lung Cell Mol Physiol 2007; 293: L720-L729.

77 Mulugeta S, Nguyen V, Russo SJ, et al. A surfactant protein C precursor protein BRICHOS domain mutation causes endoplasmic reticulum stress, proteasome dysfunction, and caspase 3 activation. Am J Respir Cell Mol Biol 2005; 32: 521-530.

78 Wang WJ, Mulugeta S, Russo SJ, et al. Deletion of exon 4 from human surfactant protein C results in aggresome formation and generation of a dominant negative. J Cell Sci 2003; 116: 683-692.

79 Beers MF, Hawkins A, Maguire JA, et al. A nonaggregating surfactant protein C mutant is misdirected to early endosomes and disrupts phospholipid recycling. Traffic 2011; 12: 1196-1210.

80 Hawkins A, Guttentag SH, Deterding R, et al. A non-BRICHOS SFTPC mutant (SP-CI73 T) linked to interstitial lung disease promotes a late block in macroautophagy disrupting cellular proteostasis and mitophagy. Am J Physiol Lung Cell Mol Physiol 2015; 308: L33-L47.

81 Lawson WE, Cheng DS, Degryse AL, et al. Endoplasmic reticulum stress enhances fibrotic remodeling in the lungs. Proc Natl Acad Sci USA 2011; 108: 10562-10567.

82 Maguire JA, Mulugeta S, Beers MF. Multiple ways to die: delineation of the unfolded protein response and apoptosis induced by Surfactant Protein C BRICHOS mutants. Int J Biochem Cell Biol 2012; 44: 101-112.

83 Maguire JA, Mulugeta S, Beers MF. Endoplasmic reticulum stress induced by surfactant protein C BRICHOS mutants promotes proinflammatory signaling by epithelial cells. Am J Respir Cell Mol Biol 2011; 44: 404-414.

84 Tanjore H, Cheng DS, Degryse AL, et al. Alveolar epithelial cells undergo epithelial-to-mesenchymal transition in response to endoplasmic reticulum stress. J Biol Chem 2011; 286: 30972-30980.

85 Maitra M, Dey M, Yuan WC, et al. Lung fibrosis-associated surfactant protein A1 and C variants induce latent transforming growth factor betal secretion in lung epithelial cells. J Biol Chem 2013; 288: 27159-27171.

86 Maitra M, Wang Y, Gerard RD, et al. Surfactant protein A2 mutations associated with pulmonary fibrosis lead to protein instability and endoplasmic reticulum stress. J Biol Chem 2010; 285: 22103-22113.

87 Lawson WE, Grant SW, Ambrosini V, et al. Genetic mutations in surfactant protein C are a rare cause of sporadic cases of IPF. Thorax 2004; 59: 977-980.

88 Markart P, Ruppert C, Wygrecka M, et al. Surfactant protein C mutations in sporadic forms of idiopathic interstitial pneumonias. Eur Respir J 2007; 29: 134-137.

89 Korfei M, Ruppert C, Mahavadi P, et al. Epithelial endoplasmic reticulum stress and apoptosis in sporadic idiopathic pulmonary fibrosis. Am J Respir Crit Care Med 2008; 178: 838-846. 

response. J Virol 2005; 79: 6890-6899.

91 Geraghty P, Wallace A, D'Armiento JM. Induction of the unfolded protein response by cigarette smoke is primarily an activating transcription factor 4-C/EBP homologous protein mediated process. Int J Chron Obstruct Pulmon Dis 2011; 6: 309-319.

92 Hengstermann A, Muller T. Endoplasmic reticulum stress induced by aqueous extracts of cigarette smoke in 3T3 cells activates the unfolded-protein-response-dependent PERK pathway of cell survival. Free Radic Biol Med 2008; 44: 1097-1107.

93 Zhao H, Yang J, Shan L, et al. Measuring the impact of cigarette smoke on the UPR. Methods Enzymol 2011; 489: $147-164$.

94 Stewart GA, Ridsdale R, Martin EP, et al. 4-Phenylbutyric acid treatment rescues trafficking and processing of a mutant surfactant protein-C. Am J Respir Cell Mol Biol 2012; 47: 324-331.

95 Crossno PF, Polosukhin VV, Blackwell TS, et al. Identification of early interstitial lung disease in an individual with genetic variations in ABCA3 and SFTPC. Chest 2010; 137: 969-973.

96 Hodgson U, Pulkkinen V, Dixon M, et al. ELMOD2 is a candidate gene for familial idiopathic pulmonary fibrosis. Am J Hum Genet 2006; 79: 149-154.

97 Pulkkinen V, Bruce S, Rintahaka J, et al. ELMOD2, a candidate gene for idiopathic pulmonary fibrosis, regulates antiviral responses. FASEB J 2010; 24: 1167-1177.

98 Bamshad MJ, Ng SB, Bigham AW, et al. Exome sequencing as a tool for Mendelian disease gene discovery. Nat Rev Genet 2011; 12: 745-755.

99 Futema M, Plagnol V, Li K, et al. Whole exome sequencing of familial hypercholesterolaemia patients negative for LDLR/APOB/PCSK9 mutations. J Med Genet 2014; 51: 537-544.

100 Renton AE, Chio A, Traynor BJ. State of play in amyotrophic lateral sclerosis genetics. Nat Neurosci 2014; 17: 17-23.

101 Hershberger RE, Hedges DJ, Morales A. Dilated cardiomyopathy: the complexity of a diverse genetic architecture. Nat Rev Cardiol 2013; 10: 531-547.

102 Esteban-Jurado C, Vila-Casadesus M, Garre P, et al. Whole-exome sequencing identifies rare pathogenic variants in new predisposition genes for familial colorectal cancer. Genet Med 2015; 17: 131-142.

$103 \mathrm{Hu} \mathrm{H}$, Roach JC, Coon H, et al. A unified test of linkage analysis and rare-variant association for analysis of pedigree sequence data. Nat Biotechnol 2014; 32: 663-669.

104 Novarino G, Fenstermaker AG, Zaki MS, et al. Exome sequencing links corticospinal motor neuron disease to common neurodegenerative disorders. Science 2014; 343: 506-511.

105 Silhan LL, Shah PD, Chambers DC, et al. Lung transplantation in telomerase mutation carriers with pulmonary fibrosis. Eur Respir J 2014; 44: 178-187.

106 Etchegary H, Pullman D, Simmonds C, et al. 'It had to be done': genetic testing decisions for arrhythmogenic right ventricular cardiomyopathy. Clin Genet 2014 [in press; DOI: 10.1111/cge.12513].

107 Schuurman AG, van der Kolk DM, Verkerk MA, et al. Maximising the efficiency of clinical screening programmes: balancing predictive genetic testing with a right not to know. Eur J Hum Genet 2015 [in press; DOI: 10.1038/ejhg.2014.269].

108 Chio A, Battistini S, Calvo A, et al. Genetic counselling in ALS: facts, uncertainties and clinical suggestions. J Neurol Neurosurg Psychiatry 2014; 85: 478-485.

109 Ghaedi M, Calle EA, Mendez JJ, et al. Human iPS cell-derived alveolar epithelium repopulates lung extracellular matrix. J Clin Invest 2013; 123: 4950-4962.

110 Yan Q, Quan Y, Sun H, et al. A site-specific genetic modification for induction of pluripotency and subsequent isolation of derived lung alveolar epithelial type II cells. Stem Cells 2014; 32: 402-413.

111 Cho SW, Kim S, Kim JM, et al. Targeted genome engineering in human cells with the Cas9 RNA-guided endonuclease. Nat Biotechnol 2013; 31: 230-232.

112 Wang H, Yang H, Shivalila CS, et al. One-step generation of mice carrying mutations in multiple genes by CRISPR/Cas-mediated genome engineering. Cell 2013; 153: 910-918. 